\title{
Noninvasive detection of skin cancers by measuring optical properties of tissues
}

Lihong V. Wang, Steven L. Jacques

Lihong V. Wang, Steven L. Jacques, "Noninvasive detection of skin cancers by measuring optical properties of tissues," Proc. SPIE 2395, Lasers in Surgery: Advanced Characterization, Therapeutics, and Systems V, (12 May 1995); doi: 10.1117/12.209142 


\title{
Non-Invasive Detection of Skin Cancers by Measuring Optical Properties of Tissues
}

\author{
Lihong Wang, Steven L. Jacques \\ Laser Biology Research Laboratory, Box 17 \\ University of Texas M. D. Anderson Cancer Center \\ 1515 Holcombe Boulevard \\ Houston, Texas, USA
}

\begin{abstract}
Skin cancer is the most frequently occurring cancer of all cancers. Each year over 500,000 new cases of skin cancer will be detected. A high percentage of skin cancers are diseases in which fatalities can be all but eliminated and morbidity reduced if detected early and treated properly. These skin lesions are distinguished generally by subjective visual inspection and their definitive diagnosis requires time-consuming expensive histopathological evaluation of excisional or incisional biopsies.

In vivo experimental evidence published in the literature has shown that cancerous skin lesions have different total diffuse reflectance spectra than non-cancerous lesions or normal skin. Therefore, cancerous skin lesions may be differentiated from non-cancerous skin lesions by comparing the optical properties of the skin lesions with those of the surrounding normal skin sites, where the optical properties of the normal skin sites are used to account for different types of skin or different areas of skin. We have demonstrated that the effect of melanin concentration on the diffuse reflectance may be removed by extrapolating the refletance at different wavelengths to an apparent pivot point. Because the concentration of melanin does not indicate maliganancy, the removal of its effect is important to avoid false detection.

The total diffuse reflectance depends on the albedo and anisotropy of tissues. Therefore, the total diffuse reflectance will remain the same as long as the anisotropy and the ratio between the absorption coefficient and the reduced scattering coefficient remain the same. Separating the absorption and scattering effects should enhance the detection sensitivity of skin cancers.
\end{abstract}

Key words: Tissue optics, skin cancers, turbid media, scattering media, absorption coefficient, reduced scattering coefficient, optical properties, Monte Carlo simulation. 


\section{INTRODUCTION}

For convenience, we include below a table of glossary relevant to this article.

\begin{tabular}{|c|c|}
\hline $\begin{array}{l}\text { Absorption coefficient } \\
{\left[\mu_{\mathrm{a}}, \mathrm{cm}^{-1}\right]}\end{array}$ & The probability of photon absorption per unit infinitesimal pathlength. \\
\hline Anisotropy $[\mathrm{g}]$ & $\begin{array}{l}\text { The average of the cosine value of the deflection angle by single } \\
\text { scattering. }\end{array}$ \\
\hline $\begin{array}{l}\text { Effective attenuation } \\
\text { coefficient }\left[\mu_{\mathrm{eff}}, \mathrm{cm}^{-1}\right]\end{array}$ & $\begin{array}{l}\text { The decay constant of light fluence far away from light source. } \mu_{\mathrm{eff}}= \\
\sqrt{3 \mu_{\mathrm{a}} \mu_{\mathrm{t}}{ }^{\prime}}=\sqrt{3 \mu_{\mathrm{a}} \mu_{\mathrm{s}}(1-\mathrm{g})} \text {. }\end{array}$ \\
\hline $\begin{array}{l}\text { Interaction coefficient } \\
{\left[\mu_{\mathrm{t}}, \mathrm{cm}^{-1}\right]}\end{array}$ & $\begin{array}{l}\text { The probability of photon interaction per unit infinitesimal pathlength, } \\
\text { where the interaction includes both absorption and scattering. } \mu_{\mathrm{t}}=\mu_{\mathrm{a}} \\
+\mu_{\mathrm{s}} \text {. Sometimes, it is also called total interaction coefficient or total } \\
\text { attenuation coefficient. }\end{array}$ \\
\hline Mean free path $[\mathrm{mfp}]$ & The mean pathlength between interactions, which is $1 / \mu_{\mathrm{t}}$. \\
\hline $\begin{array}{l}\text { Penetration depth }[\delta \\
\mathrm{cm}]\end{array}$ & $1 / \mu_{\text {eff. }}$ It represents decay constant of the light fluence in the far-field. \\
\hline $\begin{array}{l}\text { Scattering coefficient } \\
{\left[\mu_{\mathrm{s}}, \mathrm{cm}^{-1}\right]}\end{array}$ & The probability of photon scattering per unit infinitesimal pathlength. \\
\hline $\begin{array}{l}\text { Transport interaction } \\
\text { coefficient }\left[\mu_{t}^{\prime}, \mathrm{cm}^{-1}\right]\end{array}$ & $\mu_{\mathrm{t}}^{\prime}=\mu_{\mathrm{a}}+\mu_{\mathrm{s}}^{\prime}$ \\
\hline $\begin{array}{l}\text { Transport mean free } \\
\text { path }\left[\mathrm{mfp}^{\prime}\right]\end{array}$ & $1 / \mu_{\mathrm{t}}^{\prime}$ \\
\hline $\begin{array}{l}\text { Transport scattering } \\
\text { coefficient }\left[\mu_{\mathrm{s}}^{\prime}, \mathrm{cm}^{-1}\right]\end{array}$ & $\begin{array}{l}\mu_{\mathrm{s}}^{\prime}=\mu_{\mathrm{s}}(1-\mathrm{g}) . \text { Sometimes, it is also called reduced scattering } \\
\text { coefficient. }\end{array}$ \\
\hline
\end{tabular}

Skin cancer is the most frequently occurring cancer of all cancers. Each year over 500,000 new cases of skin cancer will be detected. A high percentage of skin cancers are diseases in which 
fatalities can be all but eliminated and morbidity reduced if detected early and treated properly. These skin lesions are distinguished generally by subjective visual inspection and their definitive diagnosis requires time-consuming expensive histopathological evaluation of excisional or incisional biopsies. It is highly desired to develop non-invasive in vivo diagnostics of skin cancers.

Marchesini et al. ${ }^{1}$ have shown in vivo experimental evidence that cancerous skin lesions have different optical properties than non-cancerous pigmented lesions or normal skin. They measured the total reflectance of normal skin and pigmented skin lesions ( 0.3 to $4.6 \mathrm{~mm}$ thick) at different wavelengths. Throughout the wavelength range, the reflectance values of normal and pigmented lesions are significantly different as expected. At $420 \mathrm{~nm}$, the reflectance values of melanoma lesions (cancerous) are not significantly different than those of other pigmented lesions, among which the nevi, keratosis, and lentigo are benign lesions. However, the difference becomes significant at greater wavelengths such as $650 \mathrm{~nm}$ and $780 \mathrm{~nm}$. They have achieved a sensitivity of $90.3 \%$ and a specificity of $77.4 \%$ in their discriminant analysis between nevus and melanoma.

Because the reflectance is determined by the optical properties of tissues, their results have shown that there is a difference in optical properties between cancerous skin lesions and pigmented non-cancerous lesions or normal skin sites. However, because they measured only the total reflectance, they were unable to predict whether the difference in optical properties are due to the absorption or scattering differences. The decrease in the total diffuse reflectance can be caused by either the increase of absorption or the decrease of scattering or both, and vice versa. The total diffuse reflectance alone cannot predict whether its decrease indicates an increase in high-absorbing melanin concentration or a scattering decrease due to pathological change of the tissue. The change in melanin concentration does not indicate malignancy. For example, the benign lentigo lesions have higher melanin concentration than the malignant melanoma, ${ }^{1}$ and there are both non-melanotic melanoma and highly melanotic melanoma. The scattering change should correlate better with malignancy and enhance the detection specificity.

Bigio et al have presented a similar argument. ${ }^{2}$ Cancerous tissues manifest significant architectural changes at the cellular and sub-cellular level. Cellular components that cause elastic scattering have dimensions typically on the order of visible to near-IR wavelengths. Elastic (Mie) scattering properties will be wavelength dependent. Therefore, elastic scattering properties should be a good cancer marker.

\section{METHODS}

Monte Carlo simulations have been used to compute the diffuse reflectance of a laser beam incident upon a turbid medium.3,4. Our Monte Carlo simulation package, which can run on any 
computer platforms supporting ANSI C (such as Sun, Mac, IBM PC/compatible), can be obtained from the authors (Email: lihong@laser.mda.uth.tmc.edu) or downloaded directly from our anonymous ftp site. The ftp address is laser.mda.uth.tmc.edu (129.106.60.92).

A Cartesian coordinate system is placed on the turbid medium (Fig. 1). The origin of the coordinate system is at the incident point of the laser beam that is assumed infinitely narrow for the computation.

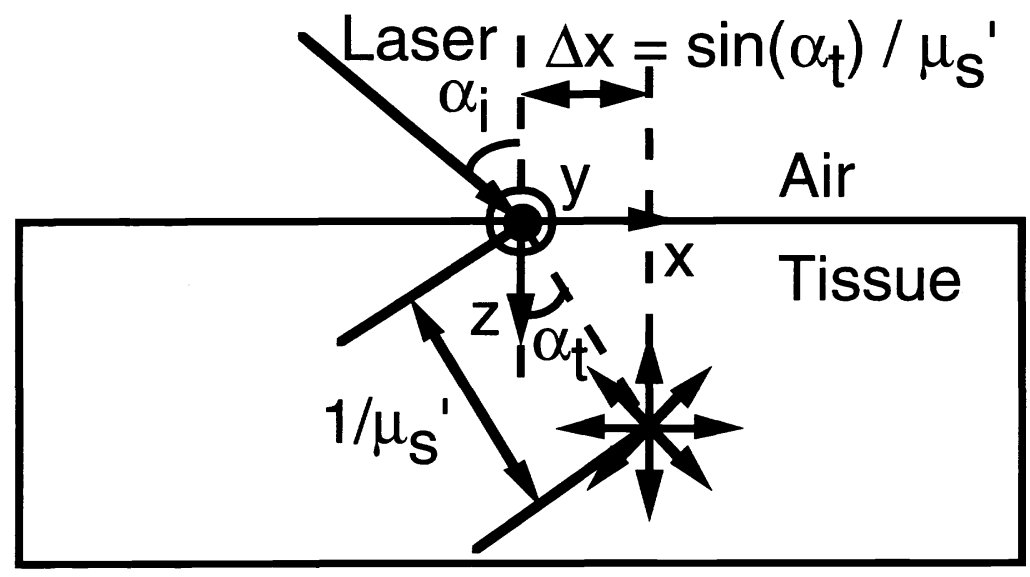

Fig. 1. Coordinate system and the lumped interaction approximation of the laser beam. In the figure, $\alpha_{i}$ is the incident angle of the laser beam, $\alpha_{t}$ is the refracted angle, $\Delta x$ is the horizontal shift of the lumped interaction site from the incident point. The y-axis point outward from the paper.

\section{RESULTS}

Are there any differences in scattering properties between cancerous lesions and noncancerous lesions? Based on Marchesini et al.'s experimental data shown as symbols in Fig. 2,1 it is found that a difference in scattering properties between malignant melanoma and benign nevi does exist. Several investigators ${ }^{5,6}$ have shown that log reflectance vs. wavelength curves of tissues containing different amount of melanin have an apparent pivot point at $837 \mathrm{~nm}$, as demonstrated with the group of radiating lines in Fig. 2. In other words, the extrapolated reflectance at $837 \mathrm{~nm}$ is independent of the melanin concentration, although the true reflectance at $837 \mathrm{~nm}$ is not equal to the extrapolated value at $837 \mathrm{~nm}$. This technique has been used to correlate the gestational age of babies with the reflectance regardless of the skin color, i.e., melanin concentration. ${ }^{7}$ Therefore, the effect of the absorption due to melanin can be removed by extrapolating the reflectance curve toward the $837 \mathrm{~nm}$ wavelength to obtain the reflectance independent of the melanin concentration. Fig. 2 shows that the diffuse reflectances after 
removing the absorption effect of melanin are $20 \%$ for the melanoma and $35 \%$ for the benign nevi. Therefore, it is indicated that the scattering properties of the melanoma and benign nevi are different assuming other absorbers in both tissues are similar. The difference in scattering properties is supported by the biological finding that the cancerous tissues are highly cellularized while the normal dermis tissues with high melanin content (nevi) are fibrous. Therefore, it is necessary to separate the absorption and scattering coefficients of the target tissue to achieve clinically significant diagnosis.

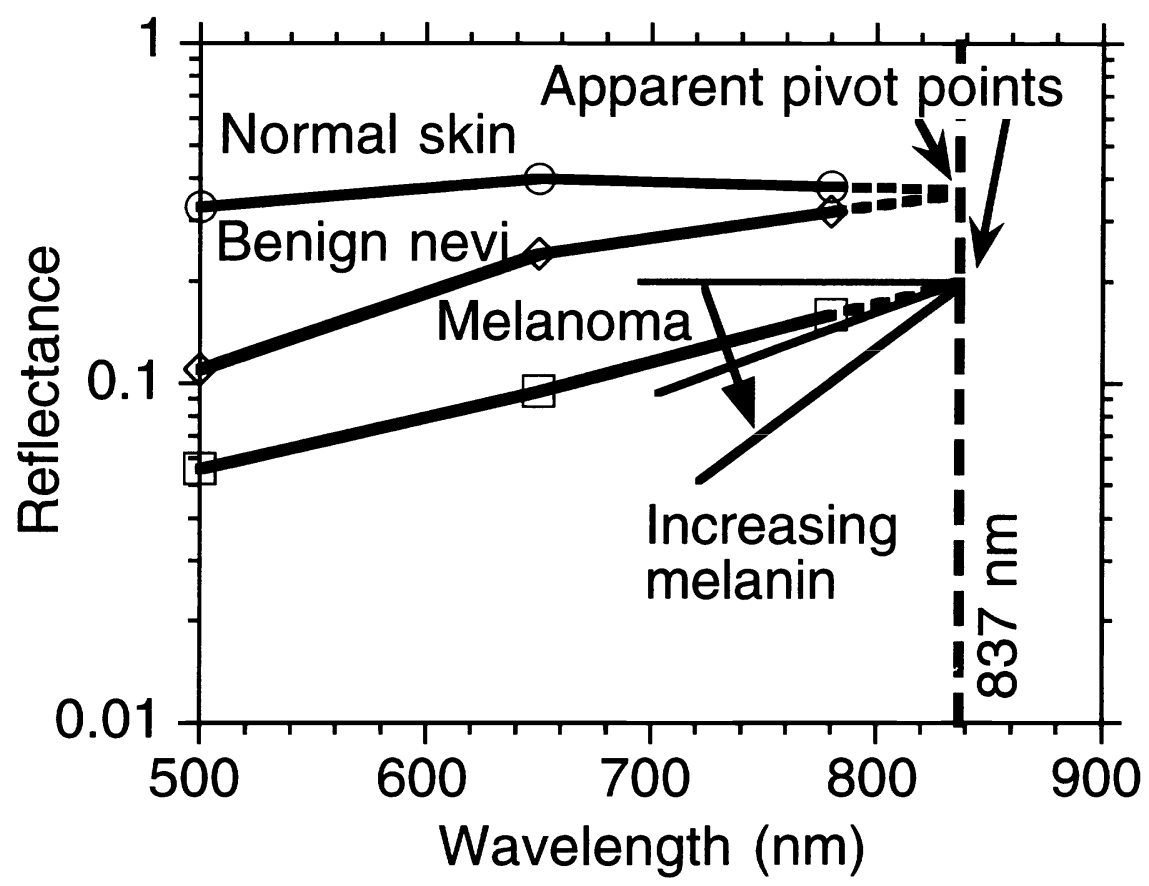

Fig. 2. The reflectance vs. the wavelength. Melanoma has lower scattering than normal skin or benign nevi.

The next question is how thick the lesion has to be to exert an enough change on the diffuse reflectance for detection. Using the Monte Carlo simulation program, we have investigated the variation in the diffuse reflectance with different thicknesses of lesion buried under skin epidermis (Fig. 3). The optical properties of the epidermis and dermis at $633 \mathrm{~nm}$ wavelength are: $\mu_{\mathrm{a}}=1.5$ $\mathrm{cm}^{-1}, \mu_{\mathrm{s}}=240 \mathrm{~cm}^{-1}, \mathrm{~g}=0.9$, and $\mu_{\mathrm{a}}=1.8 \mathrm{~cm}^{-1}, \mu_{\mathrm{s}}=440 \mathrm{~cm}^{-1}, \mathrm{~g}=0.9$, respectively. ${ }^{8}$ The reduced scattering coefficients in the literature are converted into $\mu_{\mathrm{s}}$ and $\mathrm{g}$ by assuming $\mathrm{g}$ is 0.9 . The optical properties of the lesion are tentatively set to those of the epidermis because no data is available in the literature, but are a reasonable estimate because lesions are highly cellular like the epidermis. Skin lesions may have high-absorbing melanin concentration, but this simulation was intended to show that the clinically relevant scattering property will yield a big enough change in 
the reflectance. For this setting, the diffuse reflectance drops approximately $1 \%$ for every $100-\mu \mathrm{m}$ thickness increment of the lesion, which means that the diffuse reflectance is sensitive to the thickness of the lesion when it grows into the dermis. It is interesting to see that the computed diffuse reflectance value $(\sim 40 \%)$ at $633 \mathrm{~nm}$ wavelength for normal skin matches the in vivo experimental results at $650 \mathrm{~nm} .{ }^{1}$

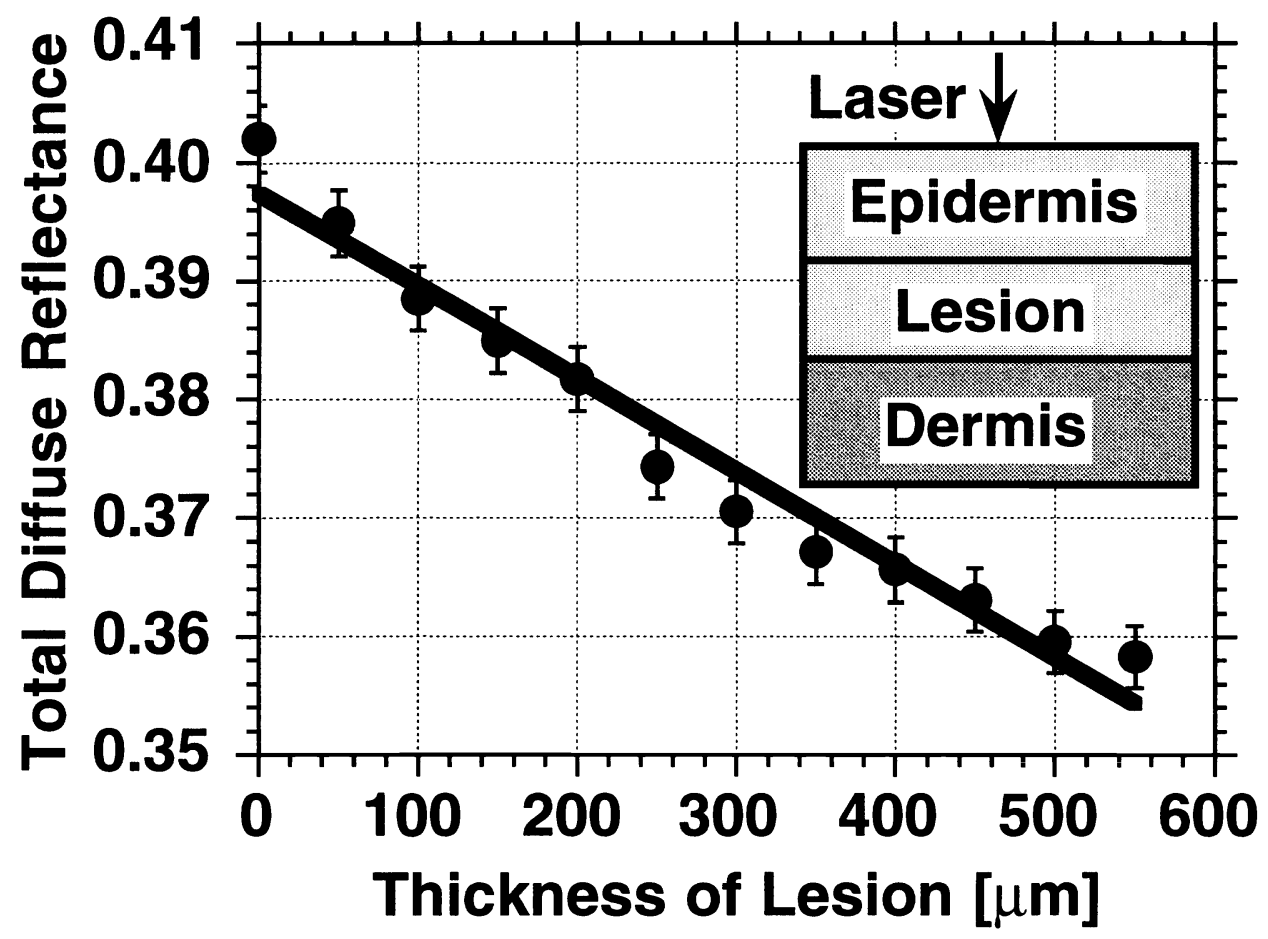

Fig. 3. Total diffuse reflectance vs. the thickness of the lesion.

To demonstrate that different combinations of absorption and scattering coefficient may yield the same total diffuse reflectance, we have assumed two tissues. The optical properties of tissues $\mathrm{A}$ are: $\mathrm{n}=1.37, \mu_{\mathrm{a}}=1 \mathrm{~cm}^{-1}, \mu_{\mathrm{s}}=100 \mathrm{~cm}^{-1}, \mathrm{~g}=0.9$. The optical properties of tissues $\mathrm{B}$ are: $\mathrm{n}=1.37, \mu_{\mathrm{a}}=2 \mathrm{~cm}^{-1}, \mu_{\mathrm{s}}=200 \mathrm{~cm}^{-1}, \mathrm{~g}=0.9$. The two hypothetical tissues have the same albedo and scattering anisotropy. The total diffuse reflectance of the two tissue computed by Monte Carlo simulations are $0.26231 \pm 0.00033$ and $0.26227 \pm 0.00034$ for tissues A and B, respectively. They are identical within the statistical error of the Monte Carlo simulation. However, the two tissues have different profiles of diffuse reflectance (Fig. 4). Therefore, we need to measure the profiles of diffuse reflectance if we want to separate the absorption and scattering effects. 


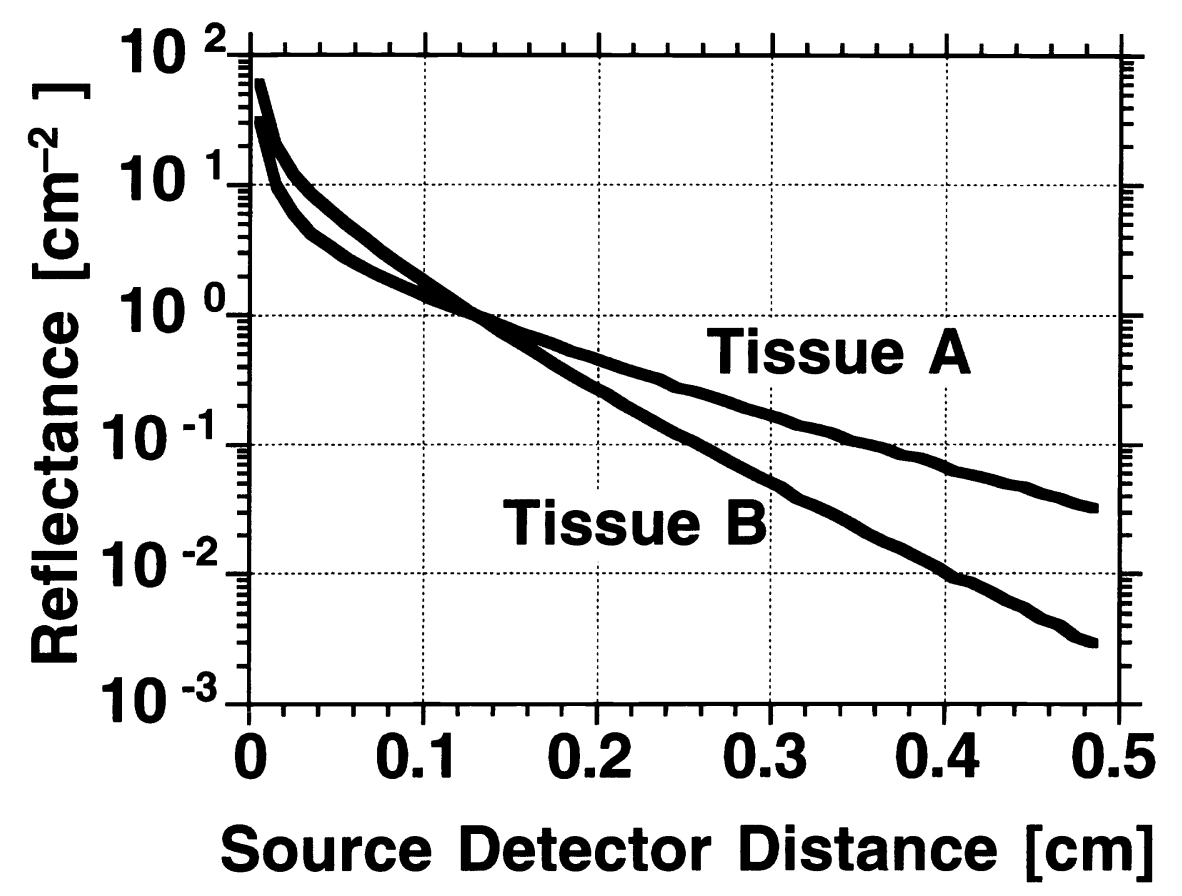

Fig. 4. Profiles of diffuse reflectance as a function of the distance between the observation points and the incident point of the laser beam.

If a laser beam is normally incident upon a semi-infinite turbid medium, the diffuse reflectance will resemble the density plot shown in Fig. 5. For symmetry reasons, the diffuse reflectance on the plot demonstrates concentric rings centered at the incident point of the laser beam. For simplicity, we will define the diffuse reflectance that is several mfp' away from the incident point of the laser beam as far diffuse reflectance. Conversely, we will define the diffuse reflectance that is within several mfp' away from the incident point of the laser beam as near diffuse reflectance.

The far diffuse reflectance becomes linear on the semi-logarithmic plot, and the slope of the diffuse reflectance is primarily determined by the penetration depth $\delta$. If one measures the relative profile of the diffuse reflectance $R(x)$, the penetration depth can be computed using a relatively simple algorithm based on the slope of the reflectance. ${ }^{9}$ If we wish to measure both absorption and reduced scattering coefficients, two independent parameters have to be determined. No simple algorithm is available to deduce the second parameter from the near diffuse reflectance. Algorithms based on Monte Carlo simulations will be time-consuming because of the stringent demand of computation time of Monte Carlo simulations. Algorithms based on diffusion theory cannot use the near diffuse reflectance because of the inaccuracy of diffusion theory in the near region. If the absolute far diffuse reflectance is measured, the diffusion theory based algorithms 
should be able to deduce the second parameter using non-linear least squares fits of the far diffuse reflectance. However, it is much easier to measure the relative profile of diffuse reflectance than to measure the absolute profile.

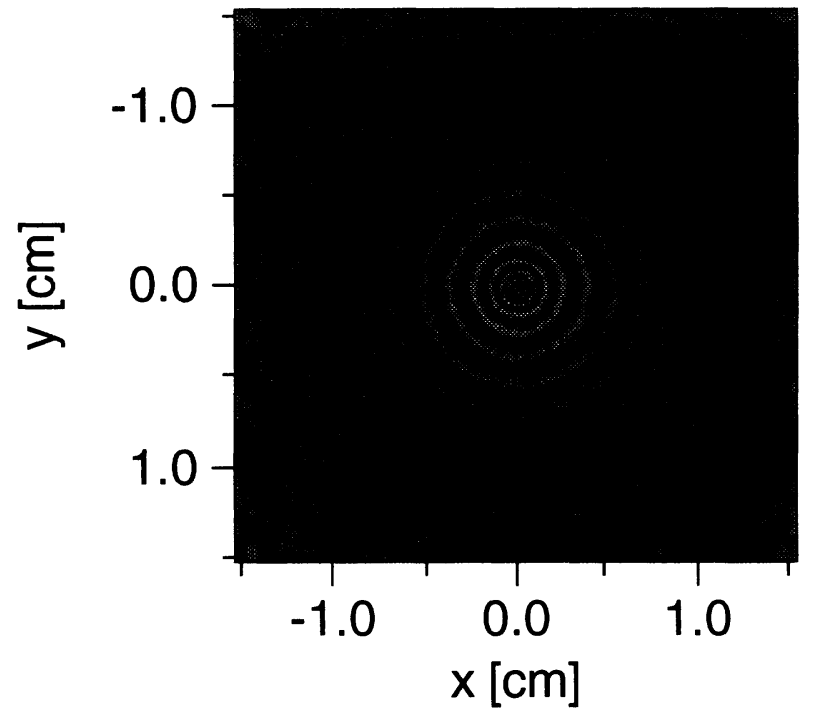
Fig. 5. 2D distribution of diffuse reflectance of a normally incident laser beam $\left(\alpha_{i}=0\right)$. The optical properties of the semi-infinite turbid medium are index of refraction $\mathrm{n}=1.33$, absorption coefficient $\mu_{\mathrm{a}}=$ $0.25 \mathrm{~cm}^{-1}, \quad$ scattering coefficient $\mu_{\mathrm{s}}=20 \mathrm{~cm}^{-1}$, anisotropy $\mathrm{g}=0.853$.

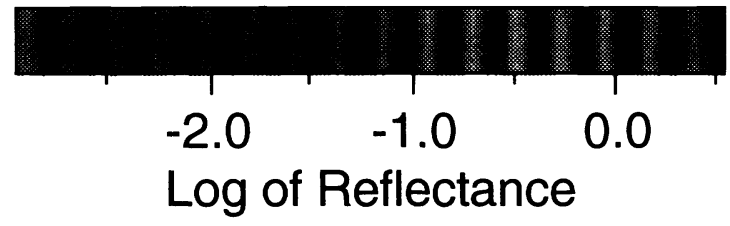

We have introduced a simple method that deduces two independent optical parameters very quickly. ${ }^{10,11}$ If a laser beam is obliquely incident upon a semi-infinite turbid medium, the diffuse reflectance will resemble the density plot shown in Fig. 6. Near the origin of the xy-plane (incident point of the laser beam), the diffuse reflectance is asymmetric. Far away from the origin, the diffuse reflectance becomes symmetric again, and there is an apparent center for the concentric rings in the far region. The apparent center is shifted toward the $+\mathrm{x}$ direction.

The diffuse reflectance of normal incidence is symmetric about the incident point, whereas the symmetry line of the far diffuse reflectance of the oblique incidence is shifted toward the $+x$ direction. The slopes of the two far diffuse reflectance curves are parallel. When the diffuse reflectance of the oblique incidence is shifted, its far diffuse reflectance matches that of normal incidence. The amount of shift is computed by

$$
\Delta x=\frac{\sin \left(\alpha_{i}\right)}{n\left(\mu_{s}^{\prime}+0.35 \mu_{\mathrm{a}}\right)},
$$


as derived in our previous publication. ${ }^{10,11}$ Eq. (1) is a refined version of the equation shown in Fig. 1.
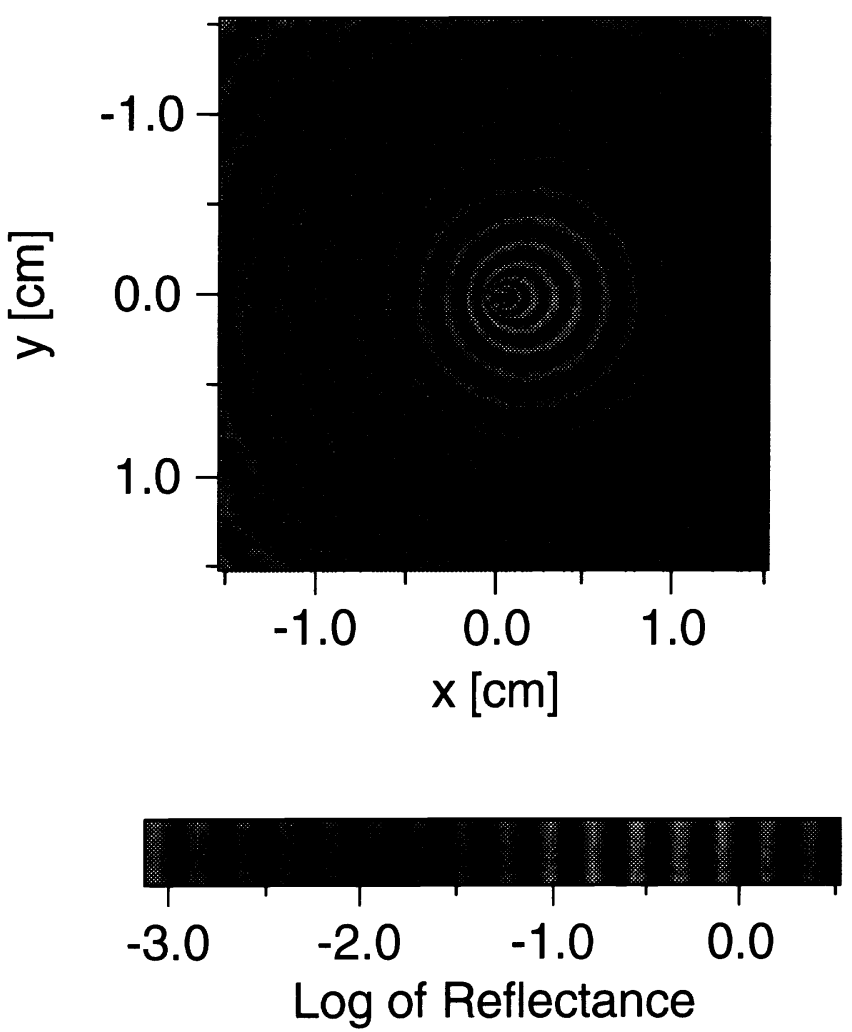

Fig. 6. 2D distribution of diffuse reflectance of an obliquely incident laser beam $\left(\alpha_{i}=45^{\circ}\right)$. The optical properties of the semi-infinite turbid medium are index of refraction $\mathrm{n}=1.33$, absorption coefficient $\mu_{\mathrm{a}}=$ $0.25 \mathrm{~cm}^{-1}$, scattering coefficient $\mu_{\mathrm{s}}=20 \mathrm{~cm}^{-1}$, anisotropy $\mathrm{g}=0.853$.

\section{CONCLUSION}

Fig. 2 shows that the contribution of melanin to the total diffuse reflectance may be removed by extrapolating the reflectance spectra to $837 \mathrm{~nm}$. The extrapolated total diffuse reflectance values of benign nevi and melanoma are different. This difference indicates that the scattering properties of the benign nevi and melanoma are different assuming other absorbers in both tissue types are similar.

The measurement of total diffuse reflectance is a sensitive tool (Fig. 3), but is independent of the individual absorption and scattering coefficients if the albedo and anisotropy are held constant. We need to separate the absorption and scattering effects to enhance the sensitivity of skin cancer detection.

We have established (Figs. 5, 6) ${ }^{10,11}$ that the shift of the apparent center of the far diffuse reflectance from the incident point of the laser beam yields the reduced scattering coefficient of the semi-infinite medium when the absorption coefficient is much less than the reduced scattering coefficient $\left(\mu_{\mathrm{a}}<<\mu_{\mathrm{s}}^{\prime}\right)$, where the computation is based on the equation shown in Fig. 1 . The 
equation was revised to Eq. (1) when $\mu_{\mathrm{a}}$ is comparable with $\mu_{\mathrm{s}}^{\prime}$. In this case, Eq. (1) can be combined with another equation deduced from the slope of the far diffuse reflectance to determined both $\mu_{\mathrm{a}}$ and $\mu_{\mathrm{s}}{ }^{\prime}$. The slope of the far diffuse reflectance of an obliquely incident laser beam is parallel with that of a normally incident laser beam upon a semi-infinite turbid medium. Therefore, the simple technique that was used to deduce the penetration depth $\delta$ in the case of normal incidence can be used similarly in the case of oblique incidence.

The oblique incidence of a laser beam upon a semi-infinite medium allows determination of two independent optical parameters using one measurement of the diffuse reflectance. Both parameters can be computed from the relative profile of the diffuse reflectance, eliminating the need for absolute measurement of the diffuse reflectance.

An optical fiber bundle, as opposed to the CCD camera system that was used in our original experiments, ${ }^{10}$ can be used to implement this technique. The optical fiber bundle approach should have the advantage of low-cost, ease of manipulation in clinical settings, and endoscopic applications. Both algorithms of deducing the optical parameters are simple and rapid. The optical fiber bundle approach may provide a real-time, non-invasive, in vivo tool for diagnosis of diseases, monitoring of physiological parameters such as hemoglobin oxygenation status, and monitoring of treatments of diseases. Clinical trials of this device for skin cancer detection have been arranged and will be reported in the future.

\section{ACKNOWLEDGMENT}

This research was supported in part by the Whitaker Foundation, Office of Naval Research grant N00014-91-J-1354, Air Force Office of Scientific Research grant F49620-93-1-0298DEF, Department of Energy grant DE-FG05-91ER61226, and National Institutes of Health grant R29HL45045.

\section{REFERENCES}

1. R. Marchesini, M. Brambilla, C. Clemente, M. Maniezzo, A. E. Sichirollo, A. Testori, D. R. Venturoli, and N. Cascinelli, "In vivo spectrometric evaluation of neoplastic and nonneoplastic skin pigmented lesions-I: reflectance measurements," Photochem. Photobiol., 53 (1), 77-84 (1991). \& "-II: discriminant analysis between nevus and melanoma," 55(4), 515-522 (1992).

2. I. J. Bigio, J. Boyer, T. M. Johnson, J. Lacey, J.R. Mourant, R. Conn, and A. Bohorfoush, "Optical diagnostics based on elastic scattering: an update of clinical 
demonstrations with the Optical Biopsy System," Proc. Soc. Photo-Opt. Instrum. Eng. 2324, 46-54 (1994).

3. L.-H. Wang and S. L. Jacques, Monte Carlo Modeling of Light Transport in Multi-layered Tissues in Standard C (The University of Texas M. D. Anderson Cancer Center, Houston, Texas, 1992).

4. L.-H. Wang, and S. L. Jacques, "Animated simulation of light transport in tissues," Proc. Soc. Photo-Opt. Instrum. Eng. 2134A, 247-254 (1994).

5. C. F. Rosen, S. L. Jacques, M. E. Stuart, R. W. Gange, "Immediate pigment darkening: visual and reflectance spectrophotometric analysis of action spectrum," Photochem. Photobiol., 51, 583-588 (1990).

6. N. Kollias, A. Baqer, "Spectroscopic characteristics of human melanin in vivo," J. Invest. Derm., 85, 38-42 (1985).

7. C. J. Lynn, I.S. Saidi, D.G. Oelberg, S.L. Jacques, "Gestational age correlates with skin reflectance in newborn infants of 24-42 weeks gestation," Biology of the Neonate (in press).

8. R. Marchesini, C. Clemente, E. Pignoli, M. Brambilla, "Optical properties of in vitro epidermis and their possible relationship with optical properties of in vivo skin," J. Photochem. Photobiol. B-Biol., 16(2), 127-140 (1992).

9. T. J. Farrell, B. C. Wilson, and M. S. Patterson, "The use of a neural network to determine tissue optical properties from spatially resolved diffuse reflectance measurements," Phys. Med. Biol. 37, 2281-2286 (1992).

10. L.-H. Wang and S. L. Jacques, "Use of a laser beam with an oblique angle of incidence to measure the reduced scattering coefficient of a turbid medium," Appl. Opt. 34, in press (1995).

11. L.-H. Wang and S. L. Jacques, "Using an obliquely incident laser beam to measure optical properties of turbid media," Proc. Soc. Photo-Opt. Instrum. Eng. 2389, in press (1995). 